\title{
Beamforming when the sound velocity is not precisely known
}

\author{
Melvin J. Hinich \\ Virginia Polytechnic Institute and State University, Blacksburg, Virginia 24061 \\ (Received 16 January 1980; accepted for publication 16 April 1980)
}

Beamforming is an integral part of most signal processing systems in active or passive sonars. The delays used to generate a beam are functions of the sound velocity, which depends on temperature, salinity, and pressure. There is a loss in array gain if the delays are incorrectly set. This will occur when the sound velocity in the water surrounding the hydrophones is different from the velocity that was used to set the delays. This paper makes two points: (1) fixed delay line sonars suffer a loss in gain when the true sound speed in the water is different from the velocity that is used to set the delays, and (2) there are signal processing techniques for two- or three-dimensional arrays that yield source bearings that are independent of the true sound velocity. These techniques require variable time delays, which can be realized using digital processing.

PACS numbers: $43.60 . \mathrm{Gk}$, 43.30.Vh

\section{INTRODUCTION}

Beamforming is an integral part of most signal processing systems in active or passive sonars, and is part of the processing used to estimate target bearings. The delays used to generate a beam are functions of the sound velocity, which depends on temperature, salinity, and pressure. Beamforming filters the received signals in "velocity space". The filter is detuned, and the beam pattern distorted, if the delays are incorrectly set. $^{2}$ This will occur when the sound velocity in the water surrounding the hydrophones is different from the velocity that was used to set the delays. Current fixed delay line sonars do not adapt to changes in sound velocity. Modern hybrid electronic beamformers, on the other hand, can be made to adapt to changes in velocity if temperature and salinity are monitored. This paper makes two points: (1) fixed delay line sonars suffer a loss in gain when the true sound speed in the water is different from the velocity that is used to set the delays, and (2) there are signal processing techniques for twoor th ree-dimensional ar rays that yield source bearings that are independent of the true sound velocity. These techniques require variable time delays, which can be realized using digital processing. The first part of this paper presents some insight to the distortion that is caused when the delays are not correctly set as a function of the true velocity. The second part presents a signal processing method for bearing estimation that is independent of the true sound velocity.

\section{THE RESPONSE OF A DETUNED BEAMFORMER}

To simplify exposition, consider a horizontal planar array of $K$ hydrophones. Let $\left(x_{k}, y_{k}\right)$ denote the coordinates of the $k$ th sensor $(k=1, \ldots, K)$ with respect to a coordinate system whose origin is at the array's center. Let $\theta_{0}$ denote the bearing of a distant source with respect to the $x$ axis (Fig. 1). Assume that the source is at the same depth as the array, so that the sound from the source propagates across the array as a plane wave with velocity $c_{0}$, the sound velocity in the water. To compute the response, let the source be a single frequency tone with unit amplitude. If the delays are set using a velocity $c$, the beamformer's power response for a look angle $\theta$ is given by

$$
\begin{aligned}
P\left(\theta_{0}, \theta\right)= & \mid \sum_{k=1}^{K} \exp i \omega_{0} \\
& \times\left.\left(\frac{x_{k} \cos \theta}{c}+\frac{y_{k} \sin \theta}{c}-\frac{x_{k} \cos \theta_{0}}{c_{0}}-\frac{y_{k} \sin \theta_{0}}{c_{0}}\right)\right|^{2},
\end{aligned}
$$

where $\omega_{0}$ is the angular frequency of the source.

If $c=c_{0}$, then $P\left(\theta_{0}, \theta_{0}\right)=K^{2}$. If $c \neq c_{0}$, it follows from (1) that $P\left(\theta_{0}, \theta_{0}\right)<K^{2}$, i.e., the response of a detuned beamformer for $\theta=\theta_{0}$ is less than the response of a tuned one.

The loss of gain for the array geometry of one of our fleet sonars cannot be presented in an unclassified paper. To obtain some insight to the loss in gain when $c$ $\neq c_{0}$, suppose that the array geometry is a square lattice with $K=N^{2}$ sensors whose interelement spacing is $d$, i.e., the sensor positions are $\{j d, l d\}$, where $j, l=0$, $\pm 1, \ldots, \pm(N-1) / 2$ (assuming $N$ odd). From (1),

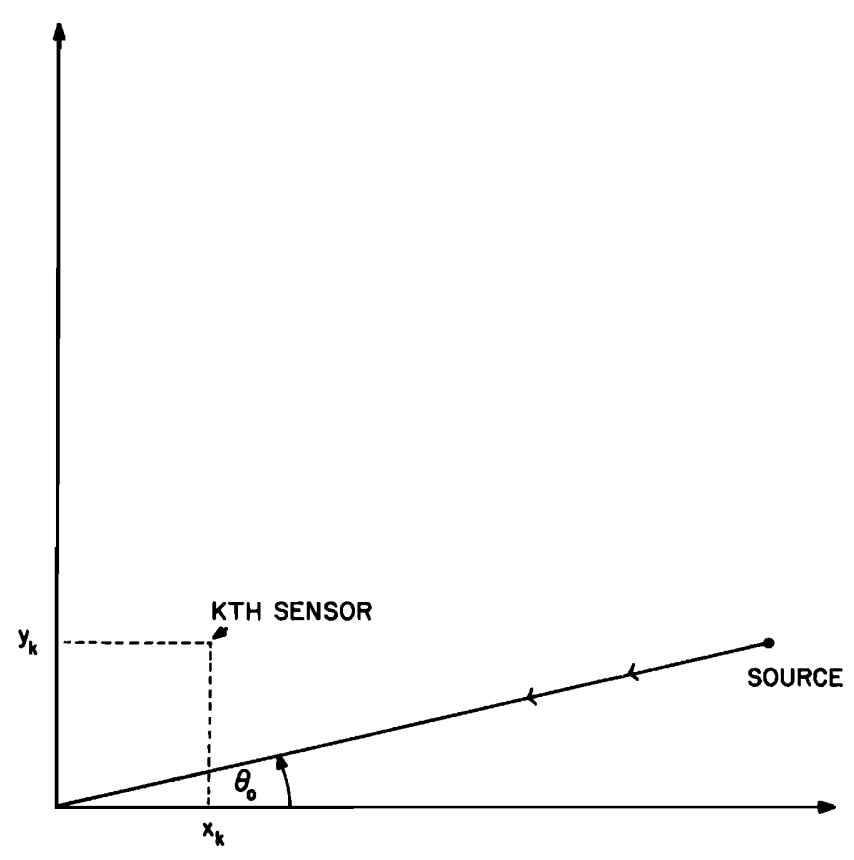

FIG. 1. Array coordinate system. 


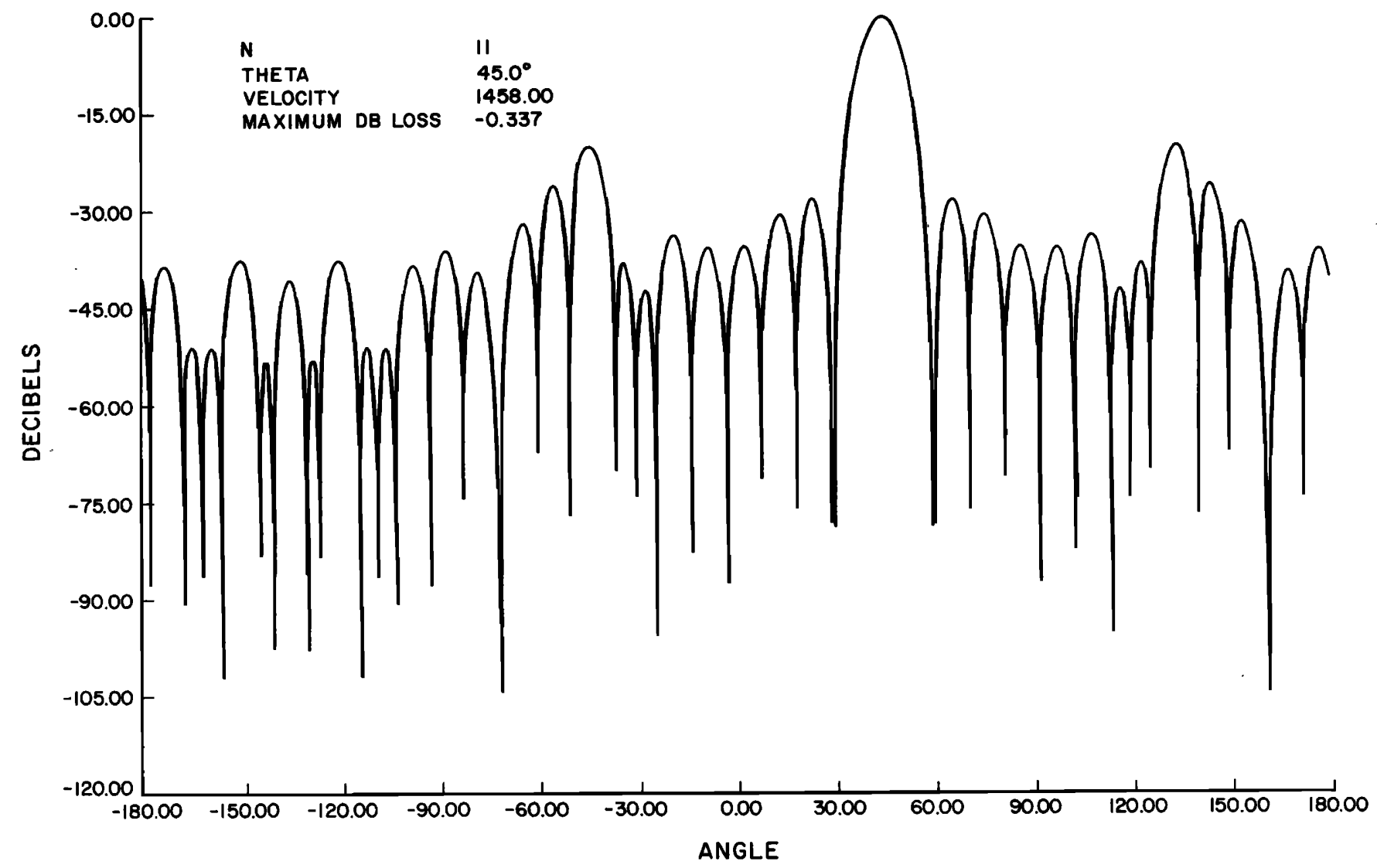

FIG. 2. Beam response when velocity is mismatched.

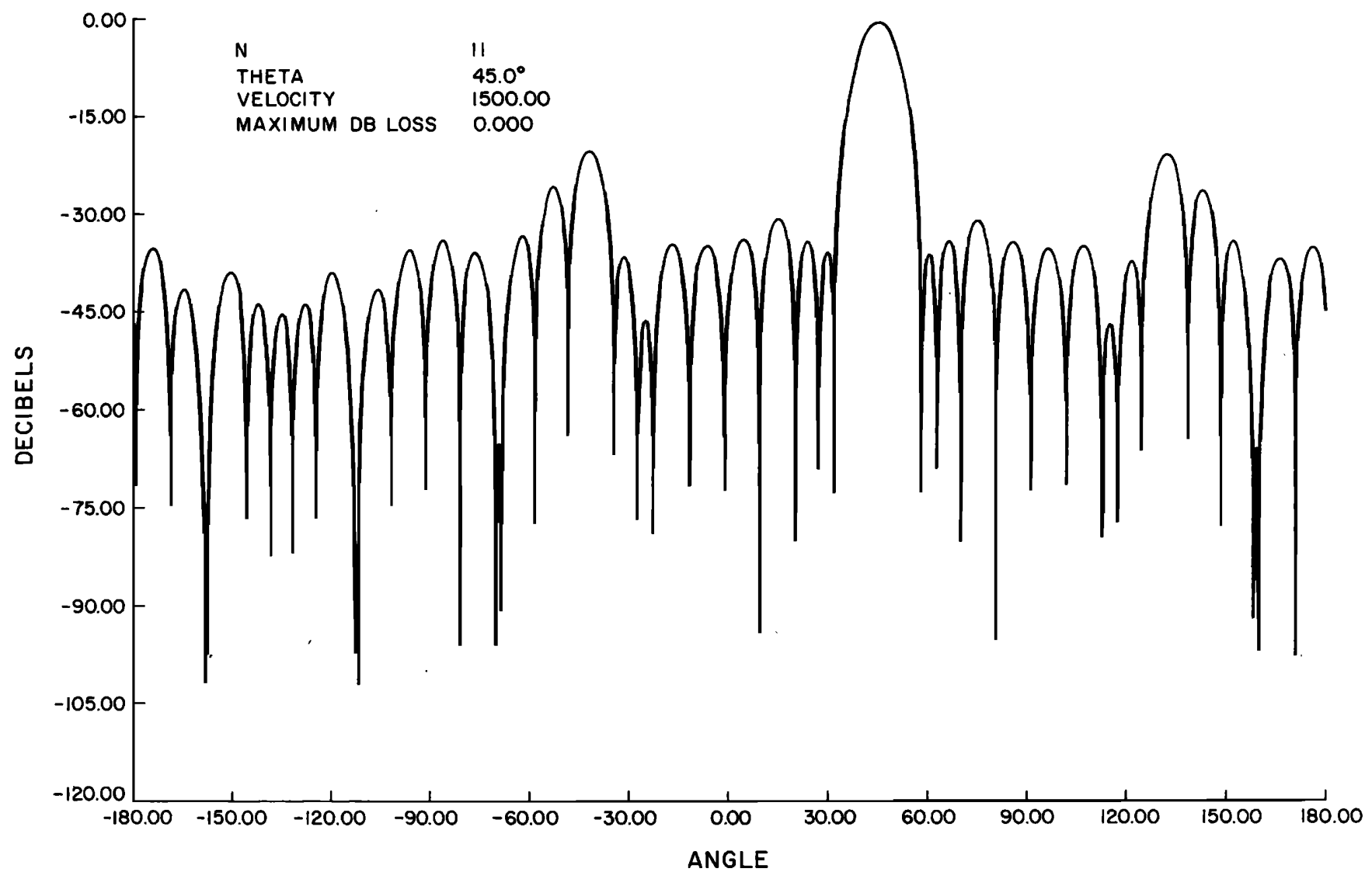

FIG. 3. Beam response when velocity is correctly set. 


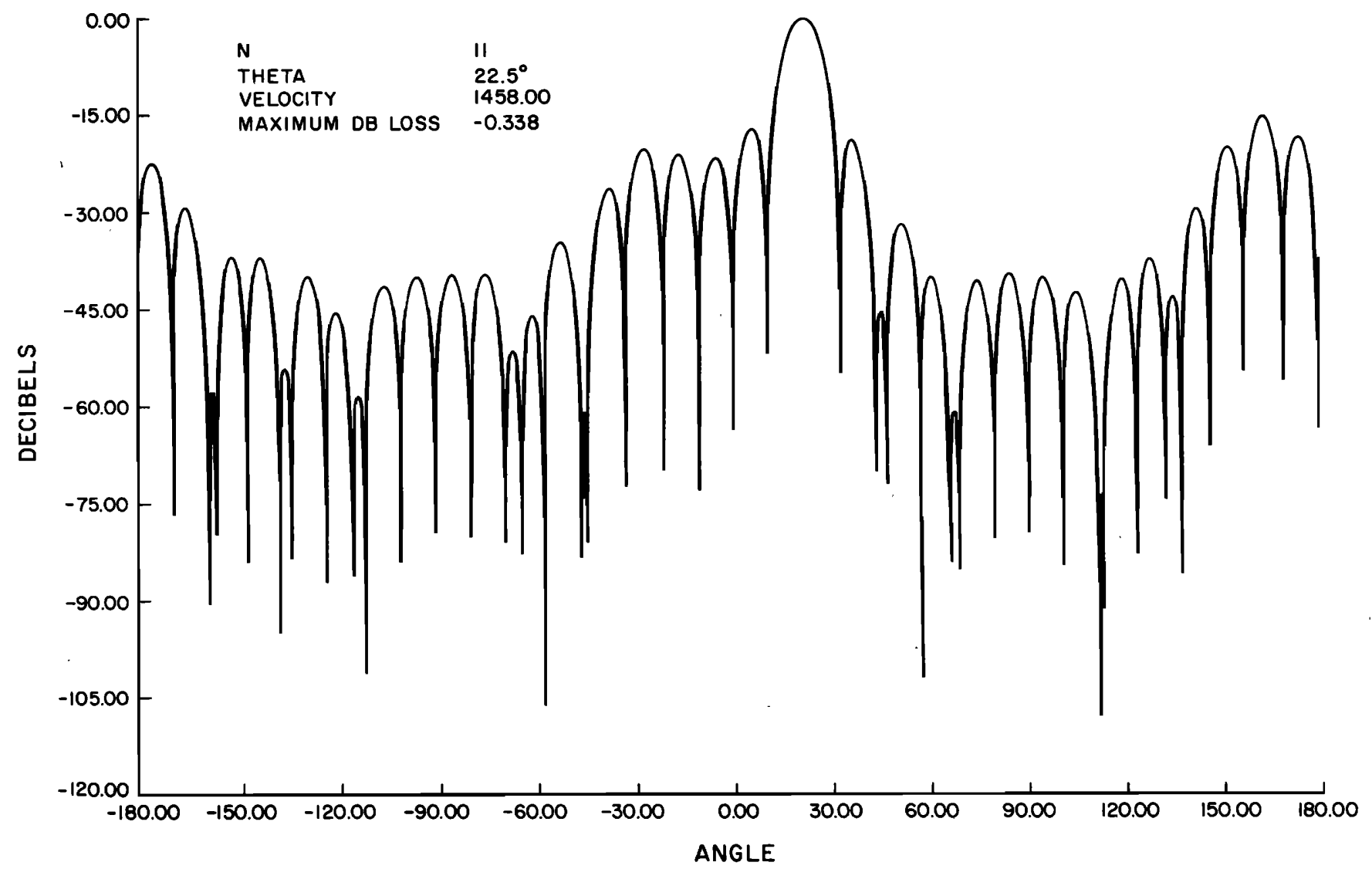

FIG. 4. Mismatched beam response for a direction of 22.5 degrees.

$$
\begin{aligned}
P\left(\theta_{0}, \theta\right)= & \frac{\sin ^{2}\left[\omega_{0}\left(c^{-1} \cos \theta-c_{0}^{-1} \cos \theta_{0}\right)(N d / 2)\right]}{\sin ^{2}\left[\omega_{0}\left(c^{-1} \cos \theta-c_{0}^{-1} \cos \theta_{0}\right)(d / 2)\right]} \\
& \times \frac{\sin ^{2}\left[\omega_{0}\left(c^{-1} \sin \theta-c_{0}^{-1} \sin \theta_{0}\right)(N d / 2)\right]}{\sin ^{2}\left[\omega_{0}\left(c^{-1} \sin \theta-c_{0}^{-1} \sin \theta_{0}\right)(d / 2)\right]}, \\
= & \frac{\sin ^{2}\left[\pi\left(c^{-1} c_{0} \cos \theta-\cos \theta_{0}\right) N d \lambda_{0}^{-1}\right]}{\sin ^{2}\left[\pi\left(c^{-1} c_{0} \cos \theta-\cos \theta_{0}\right) d \lambda_{0}^{-1}\right]} \\
& \times \frac{\sin ^{2}\left[\pi\left(c^{-1} c_{0} \sin \theta-\sin \theta_{0}\right) N d \lambda_{0}^{-1}\right]}{\sin ^{2}\left[\pi\left(c^{-1} c_{0} \sin \theta-\sin \theta_{0}\right) d \lambda_{0}^{-1}\right]},
\end{aligned}
$$

where $\lambda_{0}$ is the source wavelength. Thus

$$
\begin{aligned}
P\left(\theta_{0}, \theta_{0}\right)= & \frac{\sin ^{2}\left[\pi\left(c^{-1} c_{0}-1\right) N d \lambda_{0}^{-1} \cos \theta_{0}\right]}{\sin ^{2}\left[\pi\left(c^{-1} c_{0}-1\right) d \lambda_{0}^{-1} \cos \theta_{0}\right]} \\
& \times \frac{\sin ^{2}\left[\pi\left(c^{-1} c_{0}-1\right) N d \lambda_{0}^{-1} \sin \theta_{0}\right]}{\sin ^{2}\left[\pi\left(c^{-1} c_{0}-1\right) d \lambda_{0}^{-1} \sin \theta_{0}\right]} .
\end{aligned}
$$

The larger the array aperture, the greater the loss in gain when $c \neq c_{0}$. This implies that a large aperture linear array can be badly detuned if $c$ is not very close to $c_{0}$.

As an example, let $d \lambda_{0}^{-1}=1 / 2, N=19$, and $c=1500$ $\mathrm{m} / \mathrm{s}$. If the water temperature is $2^{\circ} \mathrm{C}$, then $c_{0}=1458$ $\mathrm{m} / \mathrm{s}^{3}$. From (3), $P\left(\theta_{0}, \theta_{0}\right) / N^{4}=0.79$ for $\theta_{0}=\pi / 4$. Since $P\left(\theta_{0}, \theta_{0}\right)=N^{4}$ if $c=c_{0}$, the loss of power is $1 \mathrm{~dB}$. The loss is also $1 \mathrm{~dB}$ for $\theta_{0}=0, \pi / 2$ and is $1.1 \mathrm{~dB}$ for $\theta_{0}$ $=\pi / 8$.

In addition to the loss in gain at $\theta=\theta_{0}$, the response is distorted. To illustrate the distortion, I plotted expression (2) as a function of $\theta_{0}$ for $\theta=\pi / 8$ and $\pi / 4$ using $N=11, c_{0}=1458 \mathrm{~m} / \mathrm{s}, c=1500 \mathrm{~m} / \mathrm{s}$, and $d=\lambda_{0} / 2$. The plot of $P\left(\theta_{0}, \pi / 4\right)$ is given in Fig. 2. As a comparison, $P\left(\theta_{0}, \pi / 4\right)$ for $c_{0}=c=1500 \mathrm{~m} / \mathrm{s}$ is given in Fig. 3. The sidelobes of the distorted response are higher than those of the undistorted response. The peak of the response is at $\theta_{0}=45^{\circ}$, but with a loss or gain of $0.34 \mathrm{~dB}$. These results are consistent with the results of Quazi and Nuttall ${ }^{2}$ for random phasing errors.

The distorted response for $\theta=\pi / 8$ is shown in Fig. 4 . The peak to sidelobe ratio is lower than that for $\theta$ $=\pi / 4$. The peak is at $\theta_{0}=22.5^{\circ}$. These results also hold when $N=19$, where the loss of gain at $\theta_{0}=\theta$ is higher.

\section{ESTIMATING BEARING INDEPENDENTLY OF VELOCITY}

Delay-and-sum processing of a planar array can be adapted to yield a bearing estimator that is independent of $c_{0}$. Let $p_{k}(t)$ denote the output from the $k$ th sensor. Assume that $p_{k}(t)$ is a plane wave $s\left(t-\alpha_{0} x_{k}-\beta_{0} y_{k}\right)$ plus stationary Gaussian noise, where $\alpha_{0}=c_{0}^{-1} \cos \theta_{0}$ and $\beta_{0}$ $=c_{0}^{-1} \sin \theta_{0}$. Sampling the channels at times $t_{1}, \ldots, t_{N}$, define the least-squares measure

$$
C(\alpha, \beta)=\sum_{j=1}^{N}\left|\sum_{k=1}^{K} p\left(t_{j}+\alpha x_{k}+\beta y_{k}\right)\right|^{2},
$$

where $\alpha$ and $\beta$ are time delays per unit distances. Let $\hat{\alpha}$ and $\hat{\beta}$ be values that jointly maximize $C(\alpha, \beta)$. Levin ${ }^{4}$ shows that, ignoring end effects, $\hat{\alpha}$ and $\hat{\beta}$ are maximum likelihood estimators of $\alpha_{0}$ and $\beta_{0}$. Using the frequency wavenumber approach to array processing, Hinich and 
Shaman $^{5}$ derive the statistical properties of maximum likelihood estimators.

Given $\hat{\alpha}$ and $\hat{\beta}$, the maximum likelihood estimator of $\theta_{0}$ is $\hat{\theta}=\tan ^{-1}(\hat{\beta} / \hat{\alpha}) .^{6}$ This estimator does not use $c_{0}$. A related robust method is given by Bennett. ${ }^{?}$

\section{ACKNOWLEDGMENTS}

This work was begun at the Naval Coastal Systems Center, Panama City, Florida under SF34371691, Sonar and Torpedo Countermeasures Block Program. The rest of the work was supported by the Office of Naval Research (Statistics and Probability Program) under contract.

${ }^{1}$ C. S. Clay, "Use of Arrays for Acoustic Transmission in a Noisy Ocean," Rev. Geophys. 4, 475-507 (1966).
${ }^{2}$ A. Quazi and A. H. Nuttall, "Effects of Random Shading, Phasing Errors and Element Failures on the Beampatterns of Line and Planar Arrays," IEEE 1979 Acoustics, Speech and Signal Processing, International Conference $(\mathrm{IEEE}$, New York, 1979), pp. 290-293.

${ }^{3}$ R. Urick, Principles of Underwater Sound (McGraw-Hill, New York, 1967), p. 94.

"M. J. Levin, "Least-Squares Array Processing for Signals of Unknown Form," Radio Electron. Eng. 29, 213-222 (1965).

${ }^{5} \mathrm{M}$. J. Hinich and P. Shaman, "Parameter Estimation for An $r$-dimensional Plane Wave Observed with Additive Independent Gaussian Errors," Ann. Math. Stat. 43, 153-169 (1972).

${ }^{6}$ C. S. Clay, M. J. Hinich, and P. Shaman, "Error Analysis of Velocity and Direction Measurements of Plane Waves Using Thick Large Aperture Arrays," J. Acoust. Soc. Am. 53, 1161-1166 (1973).

${ }^{7}$ C. M. Bennett, "A Directional Analysis of Sea Waves from Bottom Pressure Measurements," Transactions: Ocean Sciences and Engineering of the Atlantic Shelf (Marine Technology Society, Washington, DC, 1968), pp. 71-87. 